\title{
Distribution of HLA-B Locus Antigens in Patients with Psoriatic Arthritis in Bangladesh
}

\author{
Devolina Bhowmik¹, Md Ruhul Amin Miah², Shirin Tarafder², Ahmed Abu Saleh², Manash Chandra Sarker ${ }^{3}$ \\ ${ }^{1}$ Department of Microbiology, Shaheed Surawardy Medical College, Dhaka, ${ }^{2}$ Department of Microbiology and \\ Immunology, Bangabandhu Sheikh Mujib Medical University, Dhaka, ${ }^{3}$ Registrar, NITOR, Dhaka.
}

\begin{abstract}
This study was designed to investigate the distribution of HLA-B locus antigens in patients with psoriatic arthritis (PsA) in Bangladesh and identify HLA markers related to disease manifestation in PsA. HLA-B typing was carried out by polymerase chain reaction (PCR) with sequence specific primers in a group of 50 consecutive PsA patients. The reports of HLA-B locus antigens typing were collected from 50 ages and sex matched unrelated healthy donors as controls. A total of 17 HLA-B locus antigens were determined in both patients and controls. The most common antigen was B*15 (34\%) followed by B*07 (26\%), B*27 (24\%), B*38 (20\%). Human leukocyte antigens $\mathrm{B} * 07, \mathrm{~B} * 27$ and $\mathrm{B} * 38$ alleles were found to be significantly prevalent in PsA patients compared with healthy controls. We found a statistically significant association between spondylitis pattern and the presence of HLA-B*27. PsA in Bangladeshi patients seems to be associated with the presence of $\mathrm{B} * 07, \mathrm{~B} * 27$ and $\mathrm{B} * 38$ alleles.
\end{abstract}

Key words: Psoriatic arthritis (PsA), Human leukocyte antigen (HLA), Major histocompatibility complex (MHC), Polymerase chain reaction, rhematoid factor (RA)

\section{Introduction}

Psoriatic arthritis (PsA) is an inflammatory arthritis associated with psoriasis and usually seronegative in nature. It is established as a multifactorial disease resulting from a complex interplay between genetic, environmental and immunological factors. Divergent distribution of human leukocyte antigens may account for the prevalence differences of PsA in the world. Genetic factors are also important in both susceptibility to and the expression of PsA. Genetic factors are highly associated with PsA and may account for approximately $30 \%-40 \%$ of the genetic susceptibility. ${ }^{1}$ Approximately $40 \%$ of patients with PsA have a family history of psoriasis and PsA in first degree relatives. $^{2}$

There is a linkage between specific human leukocyte antigen and PsA. Genome-wide association studies (GWAS) indicate that specific class I human leukocyte antigen (HLA) genes in the major histocompatibility complex (MHC) are highly associated with PsA. ${ }^{1}$ The

\footnotetext{
Correspondence:

Dr. Devolina Bhowmik

Lecturer

Department of Microbiology

Shaheed Surawardy Medical College, Dhaka

Mobile : 01710258846, Email: drdbhowmik@yahoo.com.
}

genes that have been associated with PsA including HLA, TNF-a, IL-23R, IL-1 and killer-cell immunoglobulin like receptor (KIR) genes. ${ }^{2}$ Human leukocyte antigens are expressed in codominant manner. Alleles with the strongest PsA associations are within the HLA-B and HLA-C loci. ${ }^{3}$ These alleles, identified through advances in DNA technology, are distinguished by amino acid polymorphisms in pockets that confer the specificity to bind the side chains of particular peptides derived from different self and non-self peptides. ${ }^{4}$

Genetic association varies considerably in different races and ethnic groups due to genetic haeterogenecity There is an increased frequency of HLA- B*13, B*17, B*27, $\mathrm{B}^{*} 38, \mathrm{~B}^{*} 39, \mathrm{DR} * 4, \mathrm{DR}^{*} 7$ and $\mathrm{C}^{*} 6$ among patients with PsA in Caucasian people. ${ }^{5}$ In Indian patients HLA-B*27 is associated with PsA patients. ${ }^{6}$ The HLA alleles that are specific to PsA are HLA-B*7. B*27, B*38, and B*39 in Canadian patients. ${ }^{2}$ In Chinese population association of HLA-B*27 with PsA patients are found. ${ }^{7}$ In Spanish population there is increased frequency of HLA-B*46 and HLA-B*27. ${ }^{8}$ HLA-B*13 and HLA-B*38 alleles are found to be significantly prevalent in Israeli PsA patients. ${ }^{9}$ But data regarding association study of HLA with PsA in Bangladesh is not available.

PsA can generally affect any joint in the body. Moll and Wright (1973) describe five clinical patterns of psoriatic arthritis. ${ }^{10}$ 
1. Asymmetrical monoarticular and oligoarticular arthritis

2. Symmetrical polyarticular arthritis

3. Distal interphalangeal joint involvement

4. Arthritis mutilans

5. Axial or spondylitis.

Certain genes have been found to be more frequently associated with specific types of PsA. ${ }^{11}$ Association between distal interphalangeal joints (DIP) involvement and the presence of HLA-B*38 is found in PsA patients in Israel. ${ }^{9}$ HLA-B*27 is more frequently seen with spondylitis pattern in Chinese patients. ${ }^{11}$ Again patients who are positive for HLA-B*27 also in risk of developing axial and peripheral arthritis including distal interphalangeal joints involvement. ${ }^{12}$

Evidence suggests that the sooner PsA patients are treated, the better the prognosis and can avoid many irreversible clinical complication. ${ }^{13}$ It is established that HLA-B locus antigens are genetic markers for the development of PsA. Specific HLA molecule is also associated with specific type of clinical pattern. ${ }^{14}$

So, identification of specific HLA molecule and understanding of genetic influences is necessary to comprehend the pathophysiology of this autoimmune disease and to predict high risk patients who carry genetic susceptibility factors in general population or within families. So the aim of this study was to observe the distribution of HLA in PsA patients and to observe the relationship between HLA molecule with different clinical pattern of PsA in our geographical area.

\section{Materials and Methods}

In this population-based cross sectional study individuals from the same geographical area were compared. We selected 50 unrelated consecutive patients with PsA according to CASPAR criteria for PsA from Department of Rheumatology, Bangabandhu Sheikh Mujib Medical University (BSMMU), Dhaka. Before starting work it was approved by ethical committee of BSMMU. All patients were carefully phenotyped by rheumatologists. History of other associated autoimmune disease and past medical history were taken from previous records. Patients having other diagnosed autoimmune diseases in association with PsA were excluded for the present study. Age and sex match 50 healthy donors who came to Microbiology department of BSMMU for tissue typing for organ donation enrolled as control in this study. Persons having history of rheumatic disease or family history of PsA and other rheumatic disorders were not included as control population. Both patients and controls were Bangladeshi and came from same ethnic group. $6 \mathrm{ml}$ of blood was collected from each patient for HLA-B locus typing and for RA test. RA test was done for selection of patients according to CASPAR criteria.

\section{Detection of RF by Nephelometry:}

Patient's serum samples were tested for RF by Nephelometric system using BN ProSpec, SIEMENS, USA. The test was done according to manufacturer instructions. Evaluation was performed automatically in $\mathrm{IU} / \mathrm{ml}$ on the $\mathrm{BN}^{*}$ System. RF $>15 \mathrm{IU} / \mathrm{ml}$ was interpreted as positive. In order to ensure a valid test result controls was included in each test run.

\section{HLA-B locus typing}

Purified genomic DNA isolation was performed by DNA extraction kit according to manufacturer's instruction supplied with the PCR kit (QIAamp DNA Mini and Blood Mini Handbook 04/2010). After DNA extraction it was stored at $-20^{\circ} \mathrm{C}$ until PCR was done. Low-resolution single specific primer-polymerase chain reaction (SSP-PCR) was performed with SSP kit (DNA amplification was done by using Morgan ${ }^{\mathrm{TM}}$ HLA SSP B following manufacturer's instruction.). As low-resolution PCR reaction cannot always distinguish between closely related alleles. In this study, only serological level data were analyzed to avoid ambiguity. The amplified DNA was examined by agarose gel electrophoresis that separates the DNA fragments by size. Specific HLA-B type was determined using the worksheet (supplied along with the kit).

\section{Statistical Analysis}

All data after collection by data sheet were checked and entered in computed based SPSS 22.0 for windows software. Continuous parameters were expressed as mean \pm SD and categorical parameters as frequency and percentage. Comparisons between groups (continuous parameters) were done by unpaired t test. The frequencies of HLA-B antigens in patients and controls were compared using Chi-square and Fisher exact test. A $p$-value of $<0.05$ was considered significant.

\section{Results}

Table I shows 50 PsA patients and 50 healthy controls were enrolled in this study. Among 50 PsA patients, $27(54 \%)$ were female and $23(46 \%)$ were male with a mean age $44.60 \pm 12.16$ years (mean \pm SD). Among 50 controls, 28(56\%) were female and 22(44\%) were male with a mean age $42.46 \pm 11.5$ years (mean \pm SD). Female : male ratio was $1.2: 1$ in patients group and $1.3: 1$ in control group. 
Table-I: Distribution of study population according to their age and sex:

\begin{tabular}{cccccc}
\hline \multirow{2}{*}{ Group } & \multicolumn{3}{c}{ Sex } & & \multicolumn{2}{c}{ Age (year) } \\
\cline { 2 - 6 } & Female & Male & Female: Male & Mean \pm SD & Agerange \\
\hline Patient=50 & $27(54.0 \%)$ & $23(46.0 \%)$ & $1.2: 1$ & $44.60 \pm 12.6$ & $19-70$ \\
Control=50 & $28(56.0 \%)$ & $22(44.0 \%)$ & $1.3: 1$ & $42.46 \pm 11.5$ & $19-65$ \\
& & & & & \\
\hline
\end{tabular}

Note: Figure within parenthesis indicates percentage.

Among 50 PsA patients, symmetrical polyarthritis pattern was predominant pattern and was found in $21(42 \%)$ patients. $12(24 \%)$ PsA patients had family history of psoriasis. RA test was positive in 10 (20\%) patients (Table II).

Table-II: Clinical and demographic features of the patients with PsA $(\mathrm{n}=50)$.

\begin{tabular}{lcc}
\hline \multicolumn{1}{c}{ Features } & No. of patients & $\%$ \\
\hline Patients with asymmetrical monoarthritis & 00 & 00 \\
Patients with asymmetrical oligoarthritis & 11 & 22 \\
Patients with symmetrical polyarthritis & 21 & 42 \\
Patients with DIP joint involvement & 6 & 12 \\
Patients with spondylitis & 12 & 24 \\
Patients with arthritis mutilans & 00 & 00 \\
Patients with family history of psoriasis & 12 & 24 \\
RA test positive psoriatic arthritis patients & 10 & 20
\end{tabular}

The highest number of PsA patients was $16(32 \%)$ in the age group of 41-50 years. (Table III).

Table-III: Distribution of PsA patients according to age $(\mathrm{n}=50)$.

\begin{tabular}{ccc}
\hline Age group & No. of patients & $\%$ \\
\hline$<20$ years & 1 & 2 \\
$21-30$ years & 7 & 14 \\
$31-40$ years & 9 & 18 \\
$41-50$ years & 16 & 32 \\
$51-60$ years & 12 & 24 \\
$61-70$ years & 5 & 10 \\
Total & 50 & 100 \\
\hline
\end{tabular}

Table IV shows the frequency of HLA-B locus antigens in PsA patients and controls. A total of 17 HLA-B locus antigens were determined in both patients and controls. Among 50 PsA patients, common identified HLA-B locus antigen was $\mathrm{B}^{*} 15$ (34\%) followed by $\mathrm{B}^{*} 07$ (26\%),
B*27 (24\%), B*38 (20\%), B*52 (14\%), B*35 (12\%), B*44 (12\%), B*13 (10\%), B*40 (10\%), B*51 (10\%), B*37 (6\%), B*39 (6\%), B*57 (6\%), B*56 (4\%), B*58 (4\%), B*55(2\%). Among 50 controls, the common identified HLA-B locus antigen was B*15 (42\%) followed by B*44 (28\%), B*35 (26\%), B*40 (24\%), B*52 (14\%), B*51 (12\%), B*58 (8\%), B*57 (8\%), B*07 (8\%), B*37 $(6 \%), \mathrm{B}^{*} 38(6 \%), \mathrm{B}^{*} 08(4 \%), \mathrm{B}^{*} 27(4 \%), \mathrm{B}^{*} 56(4 \%)$, $\mathrm{B}^{*} 13(2 \%), \mathrm{B}^{*} 39(2 \%), \mathrm{B}^{*} 55(2 \%)$. The differences of frequencies of antigens of HLA-B*07, HLA-B*27 and HLA-B*38 between patient's group and control group were statistically significant and $p$ value was $0.033,0.009$ and 0.033 respectively.

Table-IV: Distribution of HLA-B locus antigens in patients and controls.

\begin{tabular}{|c|c|c|c|c|c|}
\hline $\begin{array}{c}\text { HLA-B locus } \\
\text { antigens }\end{array}$ & $\begin{array}{l}\text { Patients }(\mathrm{n}=50) \\
\text { No. }(\%)\end{array}$ & $\begin{array}{l}\text { Controls }(\mathrm{n}= \\
50) \text { No. }(\%)\end{array}$ & $P$ value & Odds ratio & $95 \% \mathrm{CI}$ \\
\hline B $* 07$ & $13(26)$ & $4(8)$ & $0.033^{\mathrm{s}}$ & 4.04 & $1.10-16.17$ \\
\hline B $* 08$ & $\mathrm{O}(\mathrm{OO})$ & 2(4) & 0.491 & 0.0 & $0.0-4.11$ \\
\hline $\mathrm{B} * 13$ & $5(10)$ & $1(2)$ & 0.203 & 5.44 & $0.58-127.9$ \\
\hline $\mathrm{B} * 15$ & $17(34)$ & $21(42)$ & 0.542 & 0.71 & $0.29-1.73$ \\
\hline $\mathrm{B} * 27$ & $12(24)$ & 2(4) & $0.009^{\mathrm{s}}$ & 7.58 & $1.46-52.38$ \\
\hline B*35 & $6(12)$ & $13(26)$ & 0.126 & 0.39 & $0.12-1.24$ \\
\hline $\mathrm{B} * 37$ & $3(6)$ & $3(6)$ & 1.00 & 1.0 & $0.15-6.63$ \\
\hline $\mathrm{B} * 38$ & $10(20)$ & $3(6)$ & $0.033^{\mathrm{s}}$ & 3.92 & $0.90-19.42$ \\
\hline B*39 & $3(6)$ & $1(2)$ & 0.609 & 3.13 & $0.27-80.96$ \\
\hline$B * 40$ & $5(10)$ & $12(24)$ & 0.110 & 0.35 & $0.10-1.21$ \\
\hline$B * 44$ & $6(12)$ & $14(28)$ & 0.08 & 0.35 & $0.11-1.11$ \\
\hline$B * 51$ & $5(10)$ & $6(12)$ & 0.749 & 0.81 & $0.20-3.32$ \\
\hline $\mathrm{B} * 52$ & $7(14)$ & $7(14)$ & 0.773 & 1.0 & $0.20-3.53$ \\
\hline $\mathrm{B} * 55$ & 1(2) & $1(2)$ & 1.000 & 1.0 & $0.0-37.84$ \\
\hline $\mathrm{B} * 56$ & 2(4) & 2(4) & 1.000 & 1.0 & $0.10-10.47$ \\
\hline B*57 & $3(6)$ & $4(8)$ & 1.000 & 0.73 & $0.12-4.18$ \\
\hline B*58 & $2(4)$ & $4(8)$ & 0.677 & 0.48 & $0.06-3.26$ \\
\hline
\end{tabular}

Note: Figure within parenthesis indicates percentage.

HLA $=$ Human leukocyte antigen, $\mathrm{s}=$ statistically significant, $\mathrm{CI}=$ confidence interval

Table $\mathrm{V}$ shows distribution of HLA-B locus antigens among clinical pattern of PsA patients. Among patients with asymmetrical oligoarthritis pattern, the most frequent identified HLA-B locus antigen was B*15(45.45\%). Patients with symmetrical polyarthritis pattern, the most frequent identified HLA-B locus antigen was $B^{*} 15(28.57 \%)$. Among patients with DIP joint involvement, the most frequent identified HLA-B locus antigen was $B^{*} 38(50 \%)$. Patients with spondylitis pattern, the most frequent identified HLA-B locus anti 
gen was $B * 27(75 \%)$. When analysis was done, a significant association was found between HLA-B*27 and spondylitis pattern $(\mathrm{p}<0.001)$.

Table-V: Distribution of HLA-B locus antigens among clinical pattern of psoriatic arthritis $(n=50)$.

\begin{tabular}{|c|c|c|c|c|c|}
\hline \multirow[b]{2}{*}{$\begin{array}{c}\text { HLA-Blocus } \\
\text { antigens }\end{array}$} & \multicolumn{5}{|c|}{ Clinical pattern } \\
\hline & $\begin{array}{l}\text { Asymmetrical } \\
\text { oligoarthritis (n } \\
=11)(\%)\end{array}$ & $\begin{array}{l}\text { Symmetrical } \\
\text { polyarthritis (n } \\
=21)(\%)\end{array}$ & $\begin{array}{c}\text { DIP } \\
\text { involvement } \\
(n=6)(\%)\end{array}$ & $\begin{array}{l}\text { Spondylitis } \\
(\mathrm{n}=12(\%)\end{array}$ & $P$ value \\
\hline$B^{*} 07(n=13)$ & $2(18.18)$ & $4(19.04)$ & $2(33.33)$ & $5(41.66)$ & 0.461 \\
\hline$B * 13(n=5)$ & $2(18.18)$ & $3(14.28)$ & $00(00)$ & $00(00)$ & 0.247 \\
\hline $\mathrm{B} * 15(\mathrm{n}=17)$ & $5(45.45)$ & $6(28.57)$ & $2(33.33)$ & $4(33.33)$ & 0.820 \\
\hline $\mathrm{B} * 27(\mathrm{n}=12)$ & $1(9.09)$ & $2(9.52)$ & $00(00)$ & $9(75)$ & $<0.001^{\mathrm{s}}$ \\
\hline$B * 35(n=6)$ & $2(18.18)$ & $2(9.52)$ & $1(16.66)$ & $1(8.33)$ & 0.850 \\
\hline$B * 37(n=3)$ & $00(00)$ & $2(9.52)$ & $1(16.66)$ & $00(00)$ & 0.370 \\
\hline $\mathrm{B} * 38(\mathrm{n}=10)$ & $1(9.09)$ & $4(19.04)$ & $3(50)$ & $2(16.66)$ & 0.232 \\
\hline$B * 39(n=3)$ & $2(18.18)$ & $00(00)$ & $1(16.66)$ & $00(00)$ & 0.113 \\
\hline$B * 40(n=5)$ & $3(27.27)$ & $2(9.52)$ & $00(00)$ & $00(00)$ & 0.129 \\
\hline$B * 44(n=6)$ & $00(00)$ & $5(23.80)$ & $00(00)$ & $1(8.33)$ & 0.154 \\
\hline$B * 51(n=5)$ & $1(9.09)$ & $4(19.04)$ & $00(00)$ & $00(00)$ & 0.270 \\
\hline$B * 52(n=7)$ & $1(9.09)$ & $4(19.04)$ & $1(16.66)$ & $1(8.33)$ & 0.796 \\
\hline$B * 55(n=1)$ & $1(9.09)$ & $00(00)$ & $00(00)$ & $00(00)$ & 0.305 \\
\hline$B * 56(n=2)$ & $00(00)$ & $2(9.52)$ & $00(00)$ & $00(00)$ & 0.411 \\
\hline$B * 57(n=3)$ & $00(00)$ & $2(9.52)$ & $00(00)$ & $1(8.33)$ & 0.645 \\
\hline$B * 58(n=2)$ & $1(9.09)$ & $00(00)$ & $1(16.66)$ & $00(00)$ & 0.202 \\
\hline
\end{tabular}

Note: Figure within parenthesis indicates percentage.

Figure within the parenthesis indicates percentage, $\mathrm{s}=$ statistically significant.

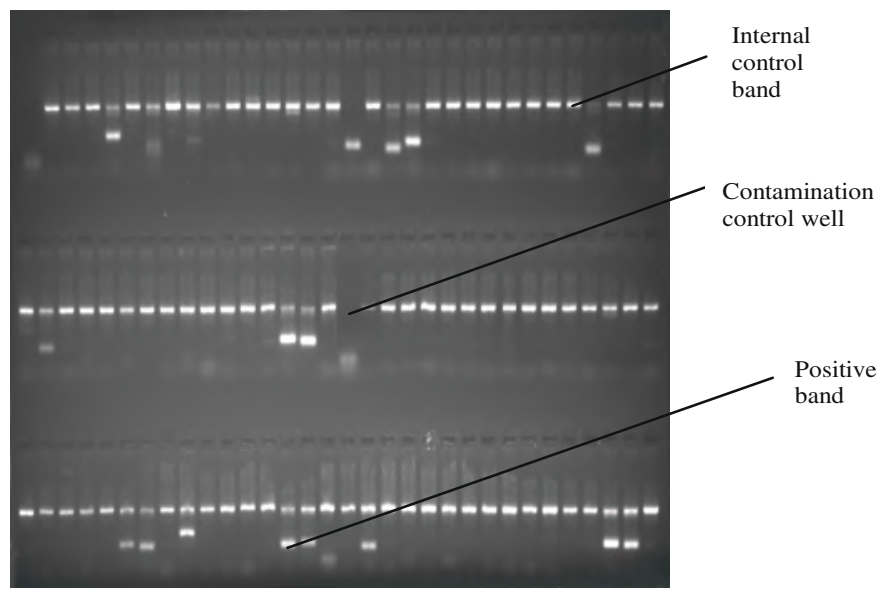

Figure-I: For HLA-B locus typing, gel electrophoresis of PCR reaction products showing internal control bands, positive control bands and contamination control well.

\section{Discussion}

Psoriatic arthritis (PsA) is a genetically complex autoimmune disease. PsA is a seronegative arthritis but recently some studies have reported that RF also present in PsA patients.

Genes in the major histocompatibility complex (MHC) or human leukocyte antigen (HLA) have been mapped to chromosome 6p21.3. They encode a series of glycoproteins that play an important part in immunological self and non-self discrimination by presenting antigens to $\mathrm{T}$ cells. ${ }^{15}$ A lot of work has been done to find out the degree and nature of association of human leukocyte antigens and PsA. Due to high degree of polymorphism within the genes of HLA, the precise genetic cause of the association has been difficult to define.

It is established that HLA-B locus antigens especially associated with PsA. ${ }^{16}$ Distributions of HLA molecules are different in different geographical areas. Due to this variation, degree of association between PsA and specific gene of the MHC also varies from one geographical area to another. Multiple reports have shown the association of PsA with HLA-B locus antigens both in Caucasian and Asian population. ${ }^{3,17}$ But study regarding association of HLA molecules with PsA in Bangladesh is not yet available. So, this study was design to see the distribution of HLA-B locus antigens in PsA patients in our geographical area.

In this study, mean age of PsA patients was $44.60 \pm 12.16$ (mean $\pm \mathrm{SD}$ ) years in between 19-70 years which is almost similar to the findings of other studies. But one study in Bangladesh, the mean age of PsA patients was $38.85 \pm 13.28$ years. ${ }^{18}$ Study on Indian patients, mean age was $42.98 \pm 12.06$ years and study on patients of Iran, the mean age of patients was $44.8 \pm 13.2$ years. ${ }^{19,20}$ Above findings indicate that PsA develops predominantly at $3^{\text {rd }}$, $4^{\text {th }}$ and $5^{\text {th }}$ decade of life.

There were $54 \%$ female and $46 \%$ male PsA patients in this study. Female: male ratio was 1.2:1 which is almost similar with the findings of study on Caucasian patients. In which female: male ratio was 1:1.21 But another study in Bangladesh and in South India, there was a different feature, female: male ratio was 1:1.74 and 1:1.7 respectively. ${ }^{18,22}$ So, in this aspect, different pictures are seen in different study. Gender-related differences have not been thoroughly explored in PsA patients. However, it is not clear whether these findings are secondary to differences in occupational physical activity, hormonal changes or other factors.

In this study, 12(24\%) patients had family history of psoriasis which is more or less similar with other study. 
Study on Bangladeshi patients, showed that 26.9\% patients had family history of psoriasis. ${ }^{18}$ There is evidence that $40 \%$ of people with PsA have a family history of psoriasis or joint disease. Children of parents with psoriasis are three times more likely to have psoriasis and are at greater risk for developing PsA than children born to parents without psoriasis. ${ }^{23}$ So, genetic factor is highly associated with PsA.

PsA is a seronegative arthritis but rheumatoid factor may be present in up to $15 \%$ of PsA patients, which could be expected given that this is a chronic inflammatory state. ${ }^{23}$ In this study $20 \%$ patients were positive for RF. Similar type of finding was found in Bangladeshi patients and in Canadian PsA patients. ${ }^{18,23}$ This result confirmed that RF is present in PsA patients but prevalence is low than rheumatoid arthritis patients because in rheumatoid arthritis, almost $100 \%$ patients are positive for RF. ${ }^{24}$ So, genetic characteristics of PsA are different from RA.

In this study, among 50 PsA patients, the most common identified HLA-B locus antigens was B*15 (34\%) followed by B*07 (26\%), B*27 (24\%), B*38 (20\%), B*52 (14\%), B*44 (12\%), B*35 (12\%), B*13 (10\%), B*40 (10\%), B*51 (10\%), B*37 (6\%), B*39 (6\%), B*57 (6\%), $\mathrm{B} * 56(4 \%), \mathrm{B} * 58(4 \%), \mathrm{B} * 55(2 \%)$. On the other hand, out of 50 controls, the common most identified HLA-B locus antigen was $\mathrm{B}^{*} 15(42 \%)$ followed by $\mathrm{B}^{*} 44(28 \%)$, B*35 (26\%), B*40 (24\%), B*52 (14\%), B*51 (12\%), $\mathrm{B} * 58(8 \%), \mathrm{B} * 57(8 \%), \mathrm{B} * 07(8 \%), \mathrm{B} * 37(6 \%), \mathrm{B} * 38$ $(6 \%), \mathrm{B}^{*} 08(4 \%), \mathrm{B}^{*} 27(4 \%), \mathrm{B} * 56(4 \%), \mathrm{B}^{*} 13(2 \%)$, B*39(2\%), B*55(2\%). A positive association of HLA-B*07, HLA-B*27 and HLA-B*38 alleles with PsA patients was observed when compared with controls ( $p=0.033,0.009$ and 0.033 respectively). Similar type of association also found in a study on PsA patients of Greater Toronto area of Canada. ${ }^{3}$ Another study in Canada found the frequency of HLA-B*27 and HLA-B*07 antigens were higher among PsA patients. ${ }^{23}$ In USA, an association was found between HLA-B*38 and PsA patients. ${ }^{25}$ In Chinese patients HLA-B*27 was associated with PsA patients. ${ }^{7}$ Study in India, found an association of HLA-B*27 with PsA patients but they did not look for other HLA-B locus antigens. ${ }^{26}$

But there are several studies that did not have similar findings with present study. A study in USA, HLA-B*13 and HLA-B*17 alleles are associated with PsA patients. ${ }^{25}$ Study on Israeli patients, showed that HLA-B*13 and B*38 alleles are associated with PsA patients but they did not find any association with HLA-B*27 allele. ${ }^{9}$
Different types of associations were reported between HLA-B locus alleles and PsA patients due to differences in geographical distribution. From above findings, it is clear that distribution of human leukocyte antigens in PsA patients is different in different geographical area because MHC polymorphism follows natural selection process.

The most common clinical pattern was symmetrical polyarthritis $(42 \%)$ followed by spondylitis pattern (24\%), asymmetrical oligoarthritis pattern $(22 \%)$ and DIP joint involvement 6(12\%). There was no patient found with asymmetrical monoarthritis and arthritis mutilans pattern. Similar finding was found in a study on patients of South India where $36.2 \%$ patients had symmetrical polyarthritis pattern. ${ }^{22}$ Similarly high noumber of PsA patients (60\%) with symmetrical polyarthritis pattern was found in Kashmir valley of India. In patients of Germany, 59\% patients had symmetrical polyarthritis pattern. ${ }^{27,28}$ So, symmetrical polyarthritis pattern was the predominant pattern both in my study and their studies.

On the contrary, Study on Bangladeshi patients, showed that asymmetrical oligoarthritis $(63.5 \%)$ pattern was the predominant pattern in PsA patients which was not similar with my study. ${ }^{18}$ Another study on Spanish patients showed that DIP (40\%) and asymmetrical oligoarthritis pattern (40\%) were the predominant pattern among PsA patients. ${ }^{29}$ So, the scenario of clinical pattern of PsA is different in different study. Over the past four decades, a number of studies have been published confirming the varied clinical patterns in PsA, partly because different definitions might have been used by individual investigators and partly due to the fact that the patterns likely change over time. If patients were suffering for long time with oligoarticular or DIP or monoarticular pattern, it tends to develop the polyarticular pattern in time. Moreover, the scenario of clinical pattern of PsA is different in different geographical area due to variation of HLA distributions in PsA patients.

In our study, in relation to clinical pattern, a significant association was found between HLA-B*27 and spondylitis pattern $(p<0.001)$ because HLA-B*27 was found more frequently in spondylitis pattern (75\%) than symmetrical polyarthritis pattern $(9.52 \%)$, asymmetrical oligoarthritis pattern $(9.09 \%)$ and DIP joint involvement $(00 \%)$. This finding is similar with the study on Chinese patients, on Caucasian patients and on South Indian patients. ${ }^{7,2,22}$ All of them found that psoriatic spondylitis pattern was associated with HLA-B*27 allele. HLA-B*27 positive PsA patients may be at risk of development of psoriatic 
spondylitis pattern in future life. It is also likely that genes out with the MHC predispose to PsA. It is further likely that a role will be found for environmental factors in PsA. There is a possibility of a complex interplay between a variety of environmental factors and genetic factors, both within and outside the MHC, determining not only susceptibility but also the individual clinical pattern of disease.

In this study, because of small sample size, study population may not represent the entire PsA patients in Bangladesh. Different study reported that along with HLA-B locus antigens, HLA-A, HLA-C, HLA-DRB1 locus antigens also associated with development of PsA. So, multicentered large population study of other HLA loci alleles and other genetic factors also required for proper identification of HLA association with PsA patients in Bangladesh.

\section{Acknowledgement}

The authors are thankful to Department of Microbiology and Immunology and Department of Rheumatology, BSMMU for their support to conduct the study. Authors are also thankful to the patients and healthy donors who were control population, for their cooperation and support that made this study possible.

\section{References}

1. Winchester R, Minevich G, Steshenko V, et al. HLA associations reveal genetic heterogeneity in psoriatic arthritis and in the psoriasis phenotype, Arthritis \& Rheumatism 2012; 64(4): 1134-1144.

2. Chandran V. Genetics of psoriasis and psoriatic arthritis, Indian Journal of Dermatology 2010; 55(2): 151-156.

3. Eder L, Chandran V, Pellet F, et al. Human leucocyte antigen risk alleles for psoriatic arthritis among patients with psoriasis, Annals of Rheumatic Disease 2012; 71: 50-55.

4. Haroon M, FitzGerald O and Winchester R. Epidemiology, genetics and management of psoriatic arthritis 2013: focus on developments of who develops the disease, its clinical features, and emerging treatment options, Psoriasis: Targets and Therapy 2013; 2: 311-13.

5. Sakkas LI, Loqueman N, Bird H, Vaugan RW, Welsh KI and Panayi GS. HLA Class II and T cell receptor gene polymorphism in psoriatic arthritis and psoriasis, Journal of Rheumatology 1990; 17: 1487-90.

6. Mishra NM and Singal V. Human Leukocyte Antigen B27 in 453 Asian Indian Patients with
Seronegative Spondyloarthropathy, Iranian Journal of Immunology 2010; 7(2).

7. Liao HT, Lin CK, Chang TY, et al. Human leukocyte antigen and clinical and demographic characteristics in psoriatic arthritis and psoriasis in Chinese patients, Journal of Rheumatology 2008; 35: 891-895.

8. Muto M, Nagai K, Mogami S, Nakano J, Sasazuki T and Asagami C. HLA antigens in Japanese patients with psoriatic arthritis, Tissue Antigens 1995; 45: 362-4.

9. Elkayam O, Segal R and Caspi D. Human leukocyte antigen distribution in Israeli patients with psoriatic arthritis, Rheumatology International 2004; 24: 93-7.

10. Moll JMH and Wright V. Psoriatic arthritis, Seminars in Arthritis and Rheumatism 1973; 3(2): 55-7821.

11. Leung YY, Tam LS, Kun EW, et al. Impact of illness and variables associated with functional impairment in Chinese patients with psoriatic arthritis, Clincal Experimental Rheumatology 2007;26: 820-6.

12. Eastmond CJ. Psoriatic arthritis. Genetics and HLA antigens, Baillieres Clinical Rheumatology 1994; 8: 263-276.

13. Haroon M, FitzGerald O and Winchester R. Epidemiology, genetics and management of psoriatic arthritis 2013: focus on developments of who develops the disease, its clinical features, and emerging treatment options, Psoriasis: Targets and Therapy 2013; 2: 311-13.

14. Queiro R, Tejon P, Coto P, et al. Clinical Differences between Men and Women with Psoriatic Arthritis: Relevance of the Analysis of Genes and Polymorphisms in the Major Histocompatibility Complex Region and of the Age at Onset of Psoriasis, Clinical and Developmental Immunology 2013; 482691: 7.

15. Bahram S, Bresnahan M, Geraghty DE, et al. A second lineage of mammalian major histocompatibility complex class I gene, Proceedings of The National Academy of Sciences of the United States of America 1994; 91: 6259-63.

16. Chandran V, Shelley B, Bullb C, et al. Human leukocyte antigen alleles and susceptibility to psoriatic arthritis, Human Immunology 2013; 74: 1333-1338.

17. Tam LS, Leung YY and Li EK. Psoriatic arthritis in Asia, Rheumatology Review 2009; 48:1473-1477.

18. Siddique MRU, Rashid MM, Wahab MA, et al. Clinical Spectra of Psoriatic Arthritis Interfacing Psoriatic Patients, Community Based Medical Journal 2012; 1(2): 26-31. 
19. Kumar R, Sharma A and Dogra S. Prevalence and clinical patterns of psoriatic arthritis patients in Indian patients with psoriasis, Indian Journal of Dermatology Venereology and Leprology 2014; 80(1): 15-23.

20. Nilay CINAR, Hatice BODUR, Filiz ESER, Ulker GUL, Muzeyyen GONUL and Isil OGUZ. The Prevalence and Characteristics of Psoriatic Arthritis in Patients with Psoriasis in a Tertiary Hospital, Archives of Rheumatology 2015; 30(1): 23-27

21. Gladman DD, Antoni C, Mease P, et al. Psoriatic arthritis: epidemiology, clinical features, course, and outcome, Annals of Rheumatic Disease 2005; 64(2): 14-7.

22. Mithun CB, Paul TA, Christina MM, et al. Clinical and immunogenetic characteristics of psoriatic arthritis: a single-center experience from South India, Internet Journal of Rheumatology and Clinical Immunology 2013; 1(1).

23. Gladman DD, Anhorn KA, Schachter RK, et al. HLA antigens in psoriatic Arthritis, Journal of Rheumatology 1986; 13: 586-92.

24. Korendowych E, Owen1 P, Ravindran J, et al. The clinical and genetic associations of anti-cyclic citrullinated peptide antibodies in psoriatic arthritis, Journal of Rheumatology 2005; 44: 1056-1060.
25. Murry C, Mann DL, Gerber LN, et al. Histocompatibility Alloantigens in Psoriasis and Psoriatic Arthritis, The Journal of Clinical Investigation 1980; 66: 670-67.

26. Mishra NM and Singal V. Human Leukocyte Antigen B27 in 453 Asian Indian Patients with Seronegative Spondyloarthropathy, Iranian Journal of Immunology 2010; 7(2).

27. Raher S, Nisa N and Arif T. The Pattern of Psoriatic Arthritis in Kashmir: A 6-Year Prospective Study, The North American Journal of Medical Sciences 2015; 7(8): 356-361.

28. Reich K, Kruger K, Mossner R, et al. Epidemiology and clinical pattern of psoriatic arthritis in Germany: a prospective interdisciplinary epidemiological study of 1,511 patients with plaque-type psoriasis, British Journal of Dermatology 2009; 160(5): 1040-7.

29. Queiro R, Gonzalez S, Lopez LC, et al. HLA-C locus alleles may modulate the clinical expression of psoriatic arthritis, Arthritis Research \& Therapy [Online] 2006; 8(6): 1-5, Available at http://arthritisresearch.com/content/8/6/R1851. 